\title{
Orthopedic Examination of Junior Female Wrestlers: A Case Study
}

\section{ISSN: 2576-8875}

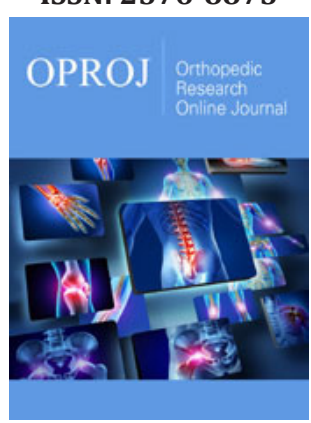

*Corresponding author: Vladimir Kurashvili MD, Federal Center for Training Sports Reserve, Russia

Submission: 価 March 03, 2021

Published: 㘹March 17, 2021

Volume 8 - Issue 1

How to cite this article: Podlivaev BA, Kurashvili VA, Gaiduk AA. Orthopedic Examination of Junior Female Wrestlers: A Case Study. Ortho Res Online J. 8(1). OPROJ. 000679. 2021. DOI: 10.31031/OPROJ.2021.08.000679

Copyright@: Kurashvili VA, This article is distributed under the terms of the Creative Commons Attribution 4.0 International License, which permits unrestricted use and redistribution provided that the original author and source are credited.

\author{
Podlivaev BA $^{1}$, Kurashvili VA ${ }^{1 *}$ and Gaiduk AA $^{2}$
}

${ }^{1}$ Federal Center for Training Sports Reserve, Russia

${ }^{2}$ Saint Petersburg State Pediatric Medical University, Russia

\begin{abstract}
Background: The aim of this study was to investigate the possible influence of female wrestling on the musculoskeletal system of junior female wrestlers.
\end{abstract}

Method: The study involved 16 freestyle junior female wrestlers. Orthopedic examination was carried out by optical topography Formetric 4D measurements.

Results: Of the 16 subjects of the sample, 12 children were orthopedically normal, 3 patients had false scoliosis and 1 has Adolescent Idiopathic Scoliosis (AIS). The results obtained revealed that contralateral side of deviation of the curve of the spine in subjects with scoliosis is statistically significant $(p=0.002)$. Furthermore, the relationship between lower midline and contralateral side of deviation of the curve of the spine in patients with false scoliosis is statistically significant $(\mathrm{p}=0.003)$.

Conclusion: The Formetric 4D provides a safe method to monitor and track the progression of postural deformities in young athletes. It can be reliably used in the surveillance of patients with AIS.

Keywords: Adolescent idiopathic scoliosis; Wrestlers; Surface topography; Formetric 4D

\section{Introduction}

Previous studies have reported sports participation as a possible risk factor for Adolescent Idiopathic Scoliosis (AIS) development. Scoliotic spinal deformity is the most serious orthopedic pathology seen in children and adolescents athletes [1,2]. The US Scoliosis Research Society has recommended annual screening of all children aged 10-14 years. The American Academy of Orthopaedic Surgeons has recommended screening girls at 11 and 13 years and screening boys at age 13 or 14 years. The American Academy of Pediatrics has recommended annual scoliosis screening with the forward bending test at routine health supervision visits [3-6].

Excessive physical activity during training in children's sports is one of the factors in the increase in the incidence of diseases among athletes. In youth sports, a specific pathology is over strain of the musculoskeletal system, which, as a rule, is the result of the combined effects of forced training loads, muscle fatigue, and adverse environmental effects. Sport and exercise are stressful by their nature, and over-stressful activity may produce injury. The lumbar spine is subjected to considerable stress during many athletic endeavors; therefore, abnormalities involving this region may cause injuries and pain among athletes because of the unique demands of the related sport [7]. In this case, chronic overstrain, stress and microtrauma occur, which disrupt the structure and function of tissues [8-10]; (Figure 1). 


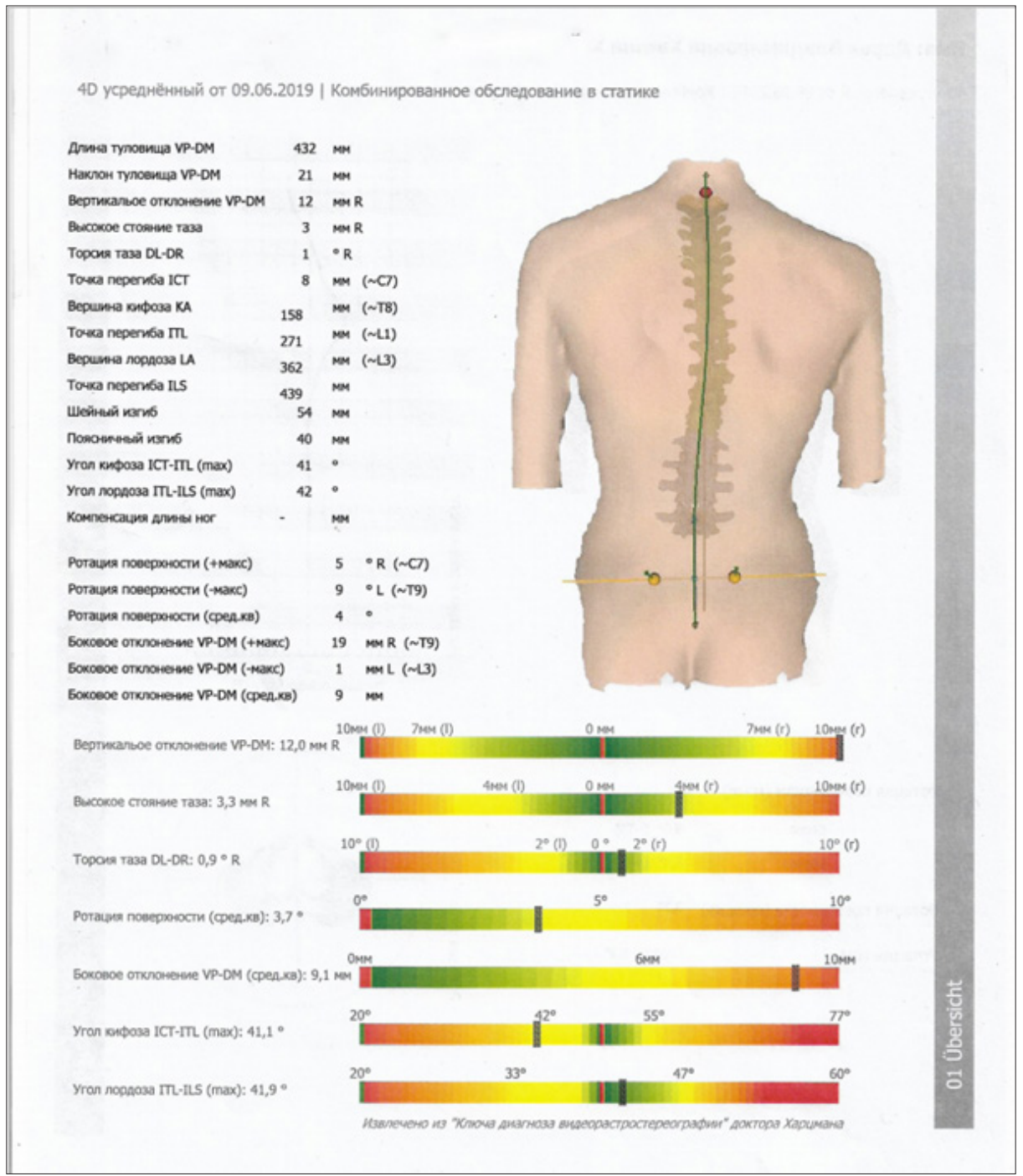

Figure 1: Combination study in frontal plane in statics.

The early start of professional sports activity, extreme physical activity (asymmetric distribution of the load on muscle groups and joints), conditions of competitive activity (special shoes, properties of the plantar surface) form and consolidate a specific motor stereotype, which determines the conditions for the development and functioning of the musculoskeletal the athlete's apparatus. Of great importance are the various conditions of sports activity, in which diseases can develop. Adolescent Idiopathic Scoliosis (AIS) is a 3D spinal deformity affecting children between the ages of 10 and 14 , without an identifiable etiology $[11,12]$.

Sports actions also include motor actions. In particular, different types of sports with repeated stresses on the spine, presenting themselves as motor engrams, can determine not only benefits deriving from muscle training, but also negative influences on the body, which affect postural structure $[13,14]$. In fact, in subjects who practice these sports at professional level, repeated stresses can lead to postural adaptations of the spine, both functional adaptive and dysfunctional [15].
According to epidemiological studies, 64 percent of young athletes engaged in sports that put a special strain on the back (gymnastics, athletics or weightlifting, wrestling, etc.) complain of chronic back pain, primarily in the lower back. Moreover, more than 70 percent of all medical prohibitions to engage in the above sports are due to dangerous pathological changes in the spine [16-18]. In the overwhelming majority, both pain syndromes and medical prohibitions on sports are caused by pathological changes in the spine, in relation to which sport acts, if not provoking, then at least a concomitant factor. In children and adolescents, pathologies such as scoliosis, juvenile osteochondrosis, spondylolysis and spondylolisthesis are most often detected $[19,20]$.

Poor posture leads to a violation of the biomechanics of the musculoskeletal system, redistributes muscle tone, which indirectly affects the further development of the child (due to the redistribution and / or asymmetry of the muscle corset, not only orthopedic, but also neurological symptoms can join). There is an opinion that in the process of development in the process 
of development against the background of impaired posture, asymmetry of internal organs can form, which in the future can manifest itself in a violation of the formation of the cardiovascular, respiratory, genitourinary, reproductive and other systems [21,22].

The current literature cites several possibilities for causative mechanisms of AIS: genetics, biomechanical growth modulation, dorsal shear forces and axial rotational instability, uncoupled spinal neuro-osseous growth, postural abnormalities and hindbrain dysfunction, motor control problems, systemic melatonin-signaling pathway deficiencies, and systemic platelet calmodulin dysfunction [23].

Topographic screening of young wrestlers was carried out using Surface Topography (ST) in surveillance of scoliosis. ST measures local deviations of a surface from a flat plane. Applying this concept to spinal deformities, ST can non-radiographically study the 3-dimensional shape of the back. One ST system, raster stereography, projects parallel white light lines onto a patient's back and analyzes line distortion with a camera. While radiography has long been considered the primary diagnostic tool for scoliosis, raster stereography may possess alternative or complementary benefits in monitoring scoliosis and other diseases. Spinal deformity was assessed by the angle of lateral asymmetry. This angle is calculated from topographic data and is analogous to the X-ray Cobb angle. The topographic parameter "rotation at the apex of the scoliotic arch" was used as a criterion for the presence of structural deformities of the spine. An analysis of the sensitivity and specificity of the topographic test in detecting structural arcs equal to $10^{\circ}$ according to Cobb and more showed that these characteristics, which determine the effectiveness of screening, depend on the level of the selected thresholds [24].

\section{Subject and Method}

Our study was carried out on a sample of 16 junior female wrestlers practicing freestyle wrestling and aged from 16.0 to 19.5 years. 16. All participants reported to a local research laboratory and completed an approved informed consent form before participating. The local institutional review board approved all aspects of the study. Various anthropometric measurements were taken (weight, height, circumferences) (Table 1).

Table 1: Anthropometric measurements in junior female wrestlers.

\begin{tabular}{|c|c|c|}
\hline Indicators & Junior Female Wrestlers (n = 16) & P \\
\hline Body length, $\mathrm{cm}$ & $165,4 \pm 0,59$ & $<0,002$ \\
\hline Body weight, $\mathrm{kg}$ & $57,4 \pm 1,02$ & $<0,001$ \\
\hline Chest circumference, cm & $84,3 \pm 0,98$ & $<0,001$ \\
\hline
\end{tabular}

Note: $\mathrm{P}$ is the level of reliability of differences in indicators.

Orthopedic examination was carried out by optical topography Formetric 4D measurements. Before being scanned, human participants completed a short medical history and a demographic questionnaire. After foot placement, the participants were asked to stand in a relaxed, natural position. In front of the participants, an adjustable fixed point was provided as a visual reference and was based on the shoulder height of the participants. Participants were instructed to focus their gaze on this fixed point during the scans to control head position. Each scan was completed in the DIERS data collection and processing software. From each of the images, 40 spine shape parameters were exported for evaluation. These parameters were sorted into three subgroups based on the clinical relatedness of the parameter. These subgroup parameters included localization and distance, trunk and pelvis imbalances, spinal reference points, spinal curve measurements, and spinal deviation. Spine shape parameters are reported in millimeters, percentage, or degrees depending on the specific parameter.

Spine shape parameter definitions adapted from DIERS formetric III 4D Manual (Created 21.06.2010, Revision grade 5) and DIERS Optical Measurement of the Spine Information for the Assessment (Version 1, Created 04.08.2009) DL left sacral dimple, DM middle point between the left and right sacral dimples, DR right sacral dimple, ICT cervical-thoracic inflection point, ILS lumbar-sacral inflection point, ITL thoracic-lumbar inflection point, KA kyphotic apex, LA lordotic apex, RMS root mean square, SP sacral point, VP vertebral prominens

\section{Result}

Of the 16 subjects of the sample, 12 children were orthopedically normal, 3 patients had false scoliosis and 1 scoliosis. The results obtained revealed that contralateral side of deviation of the curve of the spine in subjects with scoliosis is statistically significant $(p=0.002)$. Furthermore, the relationship between lower midline and contralateral side of deviation of the curve of the spine in patients with false scoliosis is statistically significant $(\mathrm{p}=0.003)$.

For 15 athletes, there were no recommendations to stop sports. But in 1 athlete during the examination, the following deviations were revealed:

\section{Frontal plane (static)}

In a natural pose there is a thoracic arch with an apex at the Th9 level with a displacement to the right by $19 \mathrm{~mm}$ (the scoliotic Cobb angle is 19 degrees), a lumbar arch with an apex at the L3 level with an offset to the left by $1 \mathrm{~mm}$ (the Cobb scoliotic angle is 11 degrees), in a natural pose vertical deviation of the spine axis to the right up to $12 \mathrm{~mm}$.

\section{Sagittal plane (static)}

In a natural position, the angle of the thoracic kyphosis is up to 41 degrees, the angle of the lumbar lordosis is 42 degrees (Figure 2). 


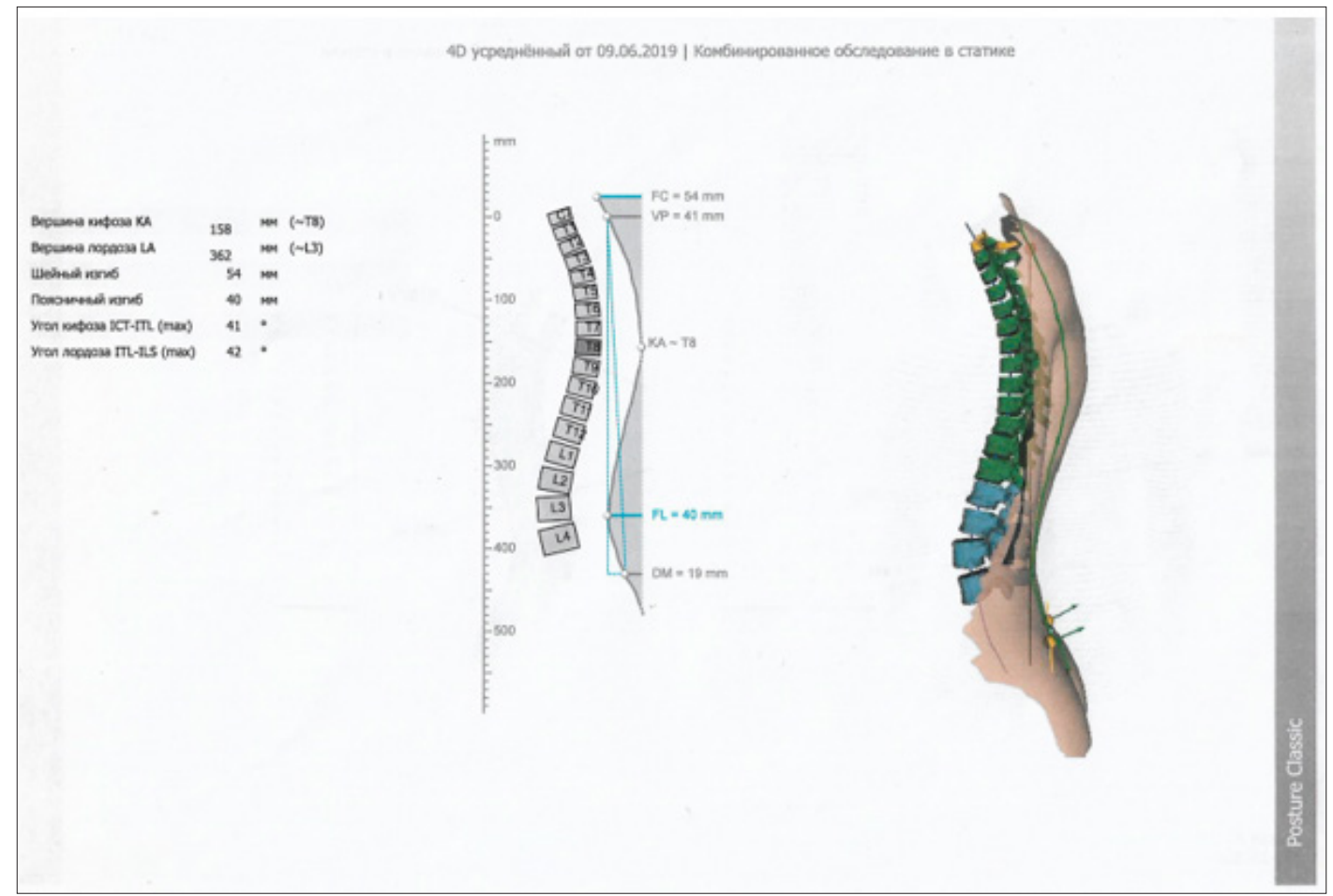

Figure 2: Image of the spine in the sagittal plane.

\section{Horizontal plane (statics)}

In a natural position, there is a rotation of the pelvis in relation to the shoulder girdle to the right by 1.3 degrees. There is an asymmetry of the paravertebral muscles in the area of the right angle of the scapula. Maximum rotation of the surface of the trunk to the left at the level of $\mathrm{C7}$, to the right at the level of Th9. The position of the pelvis in the frontal plane (statics): in a natural position, the pelvis is skewed to the left of $3 \mathrm{~mm}$.

\section{Static patient posture (body weight distribution)}

In natural posture:

A. The frontal balance of the body is slightly shifted to the right (55\%).

B. Sagittal balance of the body - no overload in any direction.

C. Preference for the right foot.

A conditional admission was issued for 6 months, with a recommendation to learn individual physical exercises for 1 month with repeated monitoring in dynamics after 5 months.

\section{Conclusion}

Screening with optical topography method can detect AIS. The basic data obtained in this study can be used to help develop a strategic exercise program for improving unilateral movement and malalignment of the spine and pelvis. Formatic 4D method might be used for the evaluation of vertebral rotation in young patients with idiopathic scoliosis, with the advantage of being easily repeatable for screening and follow-up, without any risk of exposure to ionizing radiations. Our observations show that the more technique is suitable for the screening of structural scoliosis because of its sensitivity to even small deformities and the simplicity of the technique.

Implementation of objective evaluation methods of posture, such as Formatic 4D, assumes an ever-increasing value in the field of both of the clinical and the re-educative and rehabilitative practice, since it represents an evaluation method free of ionizing radiations, rapid, scientifically recognized and easily repeatable. Based on our results, it is desirable to carry out further studies that support the use of optical topography method in the evaluation of competitive athletes.

\section{References}

1. Grayevskaya ND, Dolmatova TI (2008) Sports medicine. A course of lectures and practical sessions, p. 304.

2. Frank AS, James CM, James PD (2019) Noncontact sports participation in adolescent idiopathic scoliosis: Effects on parent-reported and patientreported outcomes. J Pediatr Orthop B 28(4): 356-361.

3. (1993) Screening for adolescent idiopathic scoliosis. Review article. US Preventive Services Task Force. JAMA 269(20): 2667-2672.

4. (1993) Screening for adolescent idiopathic scoliosis. Policy statement. US Preventive Services Task Force. JAMA 269(20): 2664-2666.

5. Richards BS, Beaty JH, Thompson GH, Willis RB (2008) Estimating the effectiveness of screening for scoliosis. Pediatrics 121(6): 1296-1297.

6. Richards BS, Vitale MG (2008) Screening for idiopathic scoliosis in adolescents. An information statements. J Bone Joint Surg Am 90(1): 195-198.

7. Elena S, Boris P, Vladimir K, Alexandr K (2020) The role of post urography on the initial stage of sports training. BIO Web of Conferences. EDP Sciences, France, pp. 1-5. 
8. Dolenko FL (2005) Sports and joints. Physical Culture and Sport, p. 288.

9. Theodoros BG, Marian HW, Stefano N, Joseph PB, Toru M, et al. (2007) SOSORT consensus paper: School screening for scoliosis. Where are we today? Scoliosis 2: 17.

10. Frank AS, James CM, James PD, Sarah E, Alexandr A, et al. (2019) Noncontact sports participation in adolescent idiopathic scoliosis: Effects on parent-reported and patient-reported outcomes. J Pediatr Orthop B 28(4): 356-361.

11. Krupatkin AI (2003) Clinical neuro Angio physiology of limbs. Scientific World, p. 327.

12. (1986) Young people's health-a challenge for society. Report of a study group on young people and health for all by the year 2000 . World Health Organization 731:1-117.

13. Ajit J, Charles EM, Steven WH, Amer F, James H, et al. (2017) Evaluation and management of adolescent idiopathic scoliosis: A review. Neurosurg Focus 43(4): E2.

14. Burwell RG (2003) Aetiology of idiopathic scoliosis: Current concepts. Pediatr Rehabil 6(3-4): 137-170.

15. Castelein RM, Dieën JH, Smit TH (2005) The role of dorsal shear forces in the pathogenesis of adolescent idiopathic scoliosis-a hypothesis. Med Hypotheses 65(3): 501-508.

16. Arthur JDL (2014) Low back pain in the adolescent athlete. Phys Med Rehabil Clin N Am 25(4): 763-788.
17. Trainor TJ, Trainor MA (2004) Etiology of low back pain in athletes. Curr Sports Med Rep 3(1): 41-46.

18. Standaert CJ (2008) Low back pain in the adolescent athlete. Phys Med Rehabil Clin N Am 19(2): 287-304.

19. Cheung JPY, Chong CHW, Cheung PWH (2019) Underarm bracing for adolescent idiopathic scoliosis leads to flatback deformity: The role of sagittal spinopelvic parameters. Bone Joint J 101-B(11): 1370-1378.

20. Pesenti S, Prost S, Blondel B, Pomero V (2020) Curve location influences spinal balance in coronal and sagittal planes but not transversal trunk motion in adolescents with idiopathic scoliosis: A prospective observational study. Eur Spine J 29(8): 1972-1980.

21. Jouve JL, Solene P, Elke V, Jean I, Lionel R, et al. (2019) Correlations linking static quantitative gait analysis parameters to radiographic parameters in adolescent idiopathic scoliosis. Orthop Traumatol Surg Res 105(3): 541-545.

22. Eardley HN, Munn Z, Cundy PJ, Gieroba TJ (2015) The effectiveness of selective thoracic fusion for treating adolescent idiopathic scoliosis: A systematic review protocol. JBI Database System Rev Implement Rep. 13(11): 4-16.

23. Frerich JM, Hertzler K, Knott P, Mardjetko S (2012) Comparison of radiographic and surface topography measurements in adolescents with idiopathic scoliosis. Open Orthop J 6: 261-265.

24. Kotwicki T, Chowanska J, Kinel E, Czaprowski D, Tomaszewski M, et al. (2013) Optimal management of idiopathic scoliosis in adolescence. Adolesc Health Med Ther 4: 59-73.

For possible submissions Click below: 